\title{
Unique role of small satellites in empowering spacecraft formation flying missions
}

\section{Peter M.B. Waswa}

The Polytechnic School, Arizona State University, 6075 S. Innovation Way West, Mesa, AZ 85212, USA

Email: pwaswa@asu.edu

\begin{abstract}
Spacecraft formation flying avails unprecedented means of conducting space missions compared to a single conventional large monolithic platform. Chiefly, formation flying enables sparse and synthesised large instrument apertures to be realised in space. Such large apertures immensely enhance the capabilities of missions conducting space astronomy, earth observation, surveillance, etc. Small satellites possess unique attributes such as modularity, small size and relatively lower costs. Consequently, this paper reviews spacecraft formation flying missions that are conducted using small satellites. In this effort, we seek to establish if small satellites play a unique role in fostering spacecraft formation flying. To that end, we endeavour to qualitatively attest the posited hypothesis that 'small satellites uniquely empower spacecraft formation flying missions'. Apart from analysing the mission architecture and system configuration, we also scrutinise systems engineering practices uniquely motivated by small spacecraft formulation requirements. The findings of this review demonstrate that small satellites possess unique characteristics that exclusively enable them to empower spacecraft formation flying missions. Moreover, we observe that the majority of the reviewed missions employ cubesats and the most common formation flying mission objective is technology demonstration.
\end{abstract}

Keywords: small spacecraft; spacecraft formation flying; cubesats.

Reference to this paper should be made as follows: Waswa, P.M.B. (2021) 'Unique role of small satellites in empowering spacecraft formation flying missions', Int. J. Space Science and Engineering, Vol. 1, No. 1, pp.55-67.

Biographical notes: Peter M.B. Waswa is a Space Systems Engineer with professional experiences in leading organisations such as NASA-JPL, Massachusetts Institute of Technology (MIT) and European Organisation for the Exploitation of Meteorological Satellites (EUMETSAT) and so on. His research interests are in formulation, implementation and sustaining of dynamical space systems and applications of space technology for sustainable development. He has authored various publications on space systems engineering and utilisation of space technology for sustainable national development. He founded http://www.spacekenya.org where he is the subject matter expert. The platform elucidates and engages a global audience on space-based technology and further advocates for sustainably integrating space technology in national development. 


\section{Introduction}

Undoubtedly, space technology is an indispensable facet of our modern way of life. We rely on space technology for communication, security, entertainment, navigation, scientific exploration, weather forecasting, etc. Since the inception of space missions - following the launch of Sputnik in 1957 (National Aeronautics and Space Administration, 1957); space mission architectures have traditionally been composed of a single large monolithic platform intended to accomplish as many tasks as possible in orbit. However, with the growth in space technology sophistication and ballooning of applications areas, new methodologies of conducting space missions became inevitable. Among these fresh mission execution approaches was flying disparate spacecraft collectively in a single mission such that the spacecraft must collaboratively accomplish the mission objectives. This approach marked the birth of distributed space systems (DSS) which encompasses spacecraft constellations, formation flying, in-orbit assembly, and most recently, spacecraft swarms.

The primary objective of this review is to scrutinise spacecraft formation flying missions that are conducted using small satellites; then, establish whether small satellites play a unique role in fostering spacecraft formation flying.

Prior to investigating if small satellites play a unique role in empowering formation flying missions, we shall define, analyse and expound on both spacecraft formation flying missions and small satellites. Notably, the words spacecraft and satellites will be used interchangeably throughout this paper and refer to a celestial man-made object purposefully fulfilling a specific mission objective(s).

The first formation flying missions were proposed in the mid-1970s to conduct a space-based interferometry mission using multiple spacecraft to synthesise a large aperture (Sholomitsky et al., 1977). Since then, a plethora of spacecraft formation flying missions has been proposed and flown utilising platforms of varied sizes and capabilities.

It is important to distinguish spacecraft formation flying from constellations. In doing so, we shall adopt the definition given by Scharf et al. (2003b). The authors define spacecraft formation flying as a set of more than one spacecraft whose states are coupled through a common control law. Moreover, at least one member must track a desired state relative to another member; and, the tracking control law must at the minimum depend upon the state of this other member. On the other hand, a constellation is a set of more than one spacecraft whose dynamic states are not coupled in any way. The change in state of any one member does not impact the state of another member.

Formation flying avails unprecedented capabilities in executing space missions key among them being sparse and synthesised large apertures. Such capabilities are employed in missions such as earth observation, communication, surveillance, space astronomy, etc. The merits of formation flying over traditional monolithic spacecraft are not however, limited to the preceding capabilities. Additionally as outlined in Scharf et al. (2003a, 2004, 2005) and Inalhan et al. (2002):

- Formation flying missions have improved overall system robustness and reliability due to the ease of incorporating redundancy and ability to distribute the payload over disparate platforms. 
- Instruments located on separate platforms in the formation can observe the same phenomena from different multiple points hence significantly increasing the knowledge gathered.

- Formation flying missions possess inherent flexibility due to the potential to reconfigure, upgrade or resize mission instruments during operation - such an option is categorically absent in monolithic platforms.

- Formation flying spacecraft are technically simpler and smaller in size compared to traditional monolithic platforms. Therefore, batch production, short lead times and relatively quicker launches are possible which translate to lower mission costs.

- Costly, long lead time-related or problematic instruments can join the fleet when ready; scalability hence further makes formations cost effective.

- Formation flying evolvability and adaptability enable uncomplicated re-sizing and re-orienting of instrument apertures. This is because, unlike conventional monoliths whose instruments apertures are constrained by the spacecraft geometric configuration, formation apertures are re-sizable through baseline variation.

- Implementation of precise formation control laws and increased autonomy implemented in formation flying eliminates extensive ground support which further improves mission efficiency and reduces costs.

While in a planetary orbital environment, spacecraft formations are subject to significant gravitational dynamics due to planetary oblateness and other environmental disturbances like aerodynamic drag, solar radiation pressure, etc. Therefore formation path generations strategies can either be based on passive relative orbits (PRO) or active relative orbits (ARO). Passive relative orbits define thrust-free periodic trajectories that utilise dynamic ambient environment to maintain spacecraft in formation. Active Relative Orbits trajectories that require open loop control to maintain their periodicity, i.e., the desired reference trajectories have to be actively attained via thrusting (Scharf et al., 2003b, 2004) and also maintain formation relative distances and attitudes. Additional control effort is required if the desired positions are located at different altitudes in order to synchronise formation members orbital periods and relative distances (Chung et al., 2016). Moreover, challenges encumbering the maturity of formation flying capabilities include highly nonlinear spacecraft orbital dynamics, collision avoidance, formation initialisation and reconfiguration, etc. As a result spacecraft formation flying still remains an active area for research and development.

Table 1 Small satellite nomenclature

\begin{tabular}{lc}
\hline Small satellite class & Mass range $(\mathrm{kg})$ \\
\hline Femtosatellites & $<0.1$ \\
Picosatellites & $0.1-1$ \\
Nanosatellites & $1-10$ \\
Microsatellites & $10-100$ \\
Minisatellites & $100-500$ \\
\hline
\end{tabular}


The term small satellites generally refers to a class of spacecrafts with a wet mass of at most $500 \mathrm{~kg}$. This grouping is further hierarchically categorised with respect to the wet mass ranges shown in Table 1. Additionally, cubesats refer to a standardised class of nanosatellites that range in size from $1 \mathrm{U}(10 \times 10 \times 10 \mathrm{~cm})$ to $6 \mathrm{U}(30 \times 20 \times 10 \mathrm{~cm})$. A standardised cubesat deployment system called a poly picosatellite orbital deployer (P-POD) is usually used to deploy cubesats in space (Chung et al., 2016).

Since the early 1990s, the space industry has witnessed a tremendous proliferation of small satellites. Not only has this surge been commensurate with small satellites areas of application, but also their capabilities continue to widen - boosting the utility of small satellites. A key substantiation behind this ebullient trend is the relatively lower costs, simplicity and short lead times that are synonymous with small satellite missions. Naturally, these characteristics appear to augur well for the propitious attributes of spacecraft formation flying bulleted above. The main objective of this research is hence to verify if this unique position occupied by small satellites potentially empower formation flying missions. Consequently, we posit the following hypothesis in this review:

- Small satellites uniquely empower spacecraft formation flying missions.

Proliferation of small satellites has not only impacted space technology applications, but has similarly altered traditional methodologies of actualising space systems (Waswa and Redkar, 2017). Development of small satellite systems has necessitated fresh, innovative and agile space systems engineering practices suitable for the simpler and lesser risk-averse platforms. Consequently, systems engineering practices targeting formation flying missions would be relatively easier to integrate into the mission development cycles of small satellites than those of traditional monolithic platforms. It is likely that this factor may not be explicitly stated in the small satellite formation missions literature under review. Therefore, our review approach shall make reasonable inferences and conclusions concerning systems engineering practices in formation flying mission development inspired by small satellites. Stimulating impact on systems engineering practices for formation flying mission is indeed a facet of empowerment that deserves acknowledgment.

Because spacecraft formation flying still remains an active area of research, our literature search targeted both proposed and flown small satellite missions that rely on formation flying to achieve mission objectives. The body of literature mustered encompassed journal articles, conference papers and mission websites.

\section{Review of missions}

To facilitate the literature review, we categorised formation flying missions into the following three classes. A brief description and illustrative examples immediately follow each classification.

\section{Earth observation missions}

Payload instruments observe given phenomena on earth, e.g., weather monitoring, remote sensing of atmosphere, land and water masses, earth imagery, etc. 


\section{Space exploration missions}

Payload instruments investigate phenomena occurring anywhere in the universe except on earth, e.g., planets, asteroids, comets, exo-planets, solar wind, cosmic rays, etc.

\section{Technology demonstration missions}

Perform on-orbit verification, validation and evaluation of technology readiness levels (TRL) and proof of concepts.

The literature search was confined to strictly small spacecraft formation flying missions according to the previously articulated definition of spacecraft formation flying. Neither constellations nor other collaborative spacecraft mission architectures were considered. This search criterion yielded the categorised publications as shown in Figure 1. The specific works are categorised as follows:

1 Earth observation missions - Wloszek et al. (2013), Peterson et al. (2008) and Gill et al. (2013)

2 space exploration missions - Underwood et al. (2015) and Plice et al. (2019)

3 technology demonstration missions - Subramanian et al. (2015), Ben-Yaacov and Gurfil (2013), Hadaegh et al. (2016), Gangestad et al. (2013), Wu et al. (2014), Griesbach et al. (2013), Leiter and Gurfil (2013), Bonin et al. (2015), Carlson and Nakamura (2000) and Cannon et al. (2018).

Figure 1 Distribution of reviewed literature

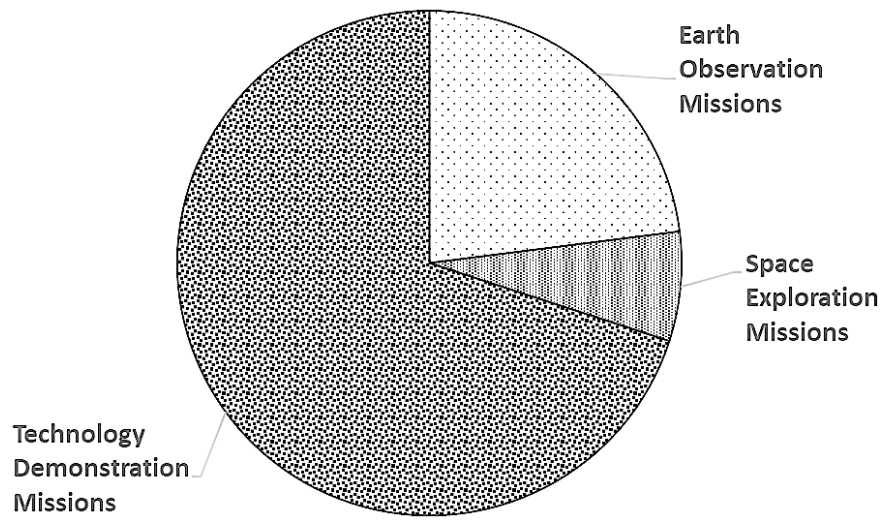

\subsection{Earth observation missions}

A formation flying mission comprising of three 6U cubesats (nanosatellites) is proposed by Wloszek et al. (2013) to investigate the troposphere in an unprecedented manner. A known time delay separating the formation spacecraft would enable the observed measurements to cooperatively construct a vertical profile of tropospheric wind enabling more accurate, reliable and longer-term weather forecasts. Moreover, to achieve global 
coverage, the formation flying mission will be part of a 12 spacecraft constellation in three orbital planes. Each formation will consist of a single spacecraft from each of the four sets in disparate orbital plane.

To provide a low cost means of augmenting proven large synthetic aperture radar (SAR), Peterson et al. (2008) present a mission of one or two small satellites in formation with a large monolithic spacecraft to constitute a multistatic interferometric synthetic aperture radar (InSAR). Such a capability has a myriad of applications that include surface deformation, digital terrain modelling, moving target detection, etc.

The formation mission in Gill et al. (2013) comprises of two nanosatellites with a scientific payload to study the variations of a number of key constituents and parameters in the lower thermosphere $(90-320 \mathrm{~km})$. In addition, a highly miniaturised propulsion system in both satellites will demonstrate formation flying capabilities. This formation is part of a large QB50 mission comprising of 50 nanosatellites from various partners around the world.

\subsection{Space exploration missions}

The first space exploration mission identified utilised a formation of two $3 \mathrm{U}$ cubesats and one $15 \mathrm{U}$ cubesat to demonstrate autonomous assembly and reconfiguration of a space telescope (Underwood et al., 2015). Validation of key technological aspects, i.e., autonomous assembly, un-docking and re-docking capabilities would enable configuration of large telescope apertures in space via formation flying. The two $3 \mathrm{U}$ nanosatellites compose the mirror component (MirrorSats) while the $15 \mathrm{U}$ microspacecraft is the Core that houses two fixed mirrors and a boom-deployed focal plane assembly. All three formation members are to be launched as a single $40 \mathrm{~kg}$ microsatellite package.

Additionally, the HelioSwarm mission (Plice et al., 2019) aims to investigate the complex three-dimensional turbulent structures that characterise the solar wind by deploying multiple co-orbiting small satellites. Once in operational orbit the individual spacecraft will use Cartesian relative motion patterns to establish baseline separations both along and across the solar wind flow direction.

\subsection{Technology demonstration missions}

Using four to six cubesats, Subramanian et al. (2015) aim to present a systems engineering-based design of formation flying mission with a nominal inter-satellite distance of $50 \mathrm{~m}$ at an altitude of $400 \mathrm{~km}$ that demonstrates requisite technology. The aim of this two-profile mission is to reconfigure into and maintain a desired post-launch geometry while avoiding inter-satellite collisions. Consequently,

1 four $3 \mathrm{U}$ cubesats maintain a tetrahedron geometry formation in low Earth orbit (LEO)

2 six cubesats autonomously reconfigure into $J_{2}$ invariant relative orbits.

In addition the article summarises the technological challenges that need to be overcome prior to successfully launching such a cubesat formation flying mission. 
The use of differential drag to achieve long-term autonomous maintenance of formations with relative distances ranging from $100 \mathrm{~m}$ to $250 \mathrm{~km}$ is proposed in Ben-Yaacov and Gurfil (2013). The formation consists of three 3U cubesats equipped with a cold-gas propulsion system, an atomic clock, an inter-satellite communication system and deployable solar panels.

A unique mission comprising a swarm of hundreds to thousands of 100-g-class femtosatellites (measuring $4 \times 4 \times 4.25 \mathrm{~cm}$ ) is proposed by Hadaegh et al. (2016). The Swarm of Silicon Wafer Integrated Femtosatellites (SWIFT) mission objective is to demonstrate realisation of sparse aperture arrays and distributed sensor networks using formation flying in LEO. The femtosatellites will host miniaturised versions of conventional spacecraft subsystems, e.g., a propulsion subsystem based on miniaturised hydrazine system, three-axis attitude and position sensors, etc. The swarm will form and maintain useful fuel-efficient three-dimensional geometric shapes.

Continued attention to swarm enabling technologies is shown by the Starling1 mission presented by Cannon et al. (2018). This mission intends to deploy a formation of three to four cubesats in LEO. Capabilities that enable affordable and scalable deep space application will be demonstrated in a series of missions involving a distributed swarm of spacecraft. Among the technology areas to be demonstrated include in-space communication, vision-based relative navigation and autonomous spacecraft reconfiguration. The mission is set for launch in early 2021.

Three 1U cubesats each with a mass of $1.2 \mathrm{~kg}$ in approximately $480 \times 780 \mathrm{~km}$ altitude orbit inclined at 65 degrees are utilised by Gangestad et al. (2013) to demonstrate formation flying control via differential aerodynamic drag initiated by cross-sectional area (wing) manipulation. Moreover, precise orbit position measurement is achieved using on-board GPS receiver with a meter-level accuracy.

A multi-objective formation flying mission is outlined in $\mathrm{Wu}$ et al. (2014) to: demonstrate autonomous formation flight, monitor sea ice in polar regions, obtain maritime information in polar regions, and validate inter-satellite communication. The formation consists of three nanosatellites - one $3 \mathrm{U}$ cubesat and two $2 \mathrm{U}$ cubesats in LEO.

Another mission to demonstrate multiple formation flying capabilities is delineated by Griesbach et al. (2013). The two 3U cubesat formation will demonstrate complex rendezvous, proximity operations (RPO), formation maintenance and docking in LEO. The nanosatellites have deployable solar panels and the missions is appropriately titled proximity operations nano-satellite flight demonstration (PONSFD).

Using two or three small satellites in LEO, Leiter and Gurfil (2013) propose to accurately determine the position of a terrestrial electromagnetic pulses emitting source, by performing measurements sequential time difference of signal arrival. Successful demonstration of this formation flying application can enable space-borne geolocation using small satellites to provide accurate tracking of a Mars rover, be a redundant navigation system in a jammed Global Satellite Navigation System (GNSS) environment, or a cost-effective system for autonomously locating distress signals.

Unlike most of the other missions, Bonin et al. (2015) report the eminent formation flying mission demonstrated by two $3 \mathrm{U}(6 \mathrm{~kg})$ cubesats in a $660 \mathrm{~km}$ LEO designated CanX-4 and CanX-5. The mission conducted in 2014 validated relative position control with an accuracy of better than $1 \mathrm{~m}$ for ten orbits in four formation configurations. Two of the formation configurations were leader-follower with relative distances of $1,000 \mathrm{~m}$ and $500 \mathrm{~m}$ apart. The other two configurations were circular projected irbits (in which 
one satellite appears to circle the other from a ground observer's standpoint) of radii $100 \mathrm{~m}$ and $50 \mathrm{~m}$.

Distinctively, Carlson and Nakamura (2000) propose a tethered approach to maintain relative spacecraft position in a formation. The two-nanosatellite mission intends to develop and test space tether deployment and operation technologies using small satellites. Additionally, the mission will test inter-satellite communication technologies. This mission jointly proposed by Kyushu University, Arizona State University and Santa Clara University will deploy a $2 \mathrm{~km}$ tether then collaborate to maintain the formation at an altitude of $400-1,200 \mathrm{~km}$.

\section{Findings and future research direction}

\subsection{Findings}

To attest our previously postulated hypothesis, we carefully scrutinised small satellites' attributes and capabilities that uniquely enabled the reviewed missions; such that, the particular mission would have been considerably untenable if exclusively executed via traditional monolithic platforms.

According to Wloszek et al. (2013), small spacecraft enable the flight proven miniaturised Fourier Transform Spectrometer (FTS) to be flown on a mission. On the other hand, an equivalent (though unproven) Doppler Lidar on a conventional monolithic spacecraft would cost $\$ 650$ million - not to mention the additional costs related to long lead times and TRL maturation. Clearly, the size, cost and amenability to instrumentation of cubesats uniquely make this mission possible. Similarly, in Peterson et al. (2008), the need for high value-to-cost factor of augmenting existing SAR missions with interferometric capabilities can only be accomplished via small satellites. Moreover, due to their size and cost, a network of cubesats is the exclusive means to realistically obtain in-situ measurements in the lower thermosphere according to the mission by Gill et al. (2013). The HelioSwarm mission (Plice et al., 2019) requires contemporaneous spatially and temporally distributed measurements to study the complex three-dimensional structures of solar wind. This objective is rendered economically and technically feasible because the nine spacecraft required are small satellite. Passive formation control using differential drag outlined in the missions by Ben-Yaacov and Gurfil (2013) and Gangestad et al. (2013) is largely feasible to implement solely using small satellites. This is because a slight difference in the cross sectional area of two similar small spacecraft corresponds to a significant change in their aerodynamic drag properties. Consequently, formation control via differential drag requires relatively less effort to implement using small satellites.

Apart from solely possessing the capability to either host an instrument payload, or be fundamentally an integral part of the instrument functionality; other attributes render small satellites irreplaceable in certain missions. Such features are related to spacecraft mass, power, geometric dimensions, etc. For instance, the illustrious formation flying mission in Bonin et al. (2015) imputes its success to cubesats' ability to innocuously piggy-back on other missions' launch opportunities. This would be inconceivable with the behemoth monolithic platforms. Comparably, the mission requirements stated in Subramanian et al. (2015) can only be met by small satellites, i.e., mass $<4 \mathrm{~kg}$, energy $<$ 100 watt-hours, power $<1.5 \mathrm{~W}$. Likewise, a swarm mission proposed by Hadaegh et al. 
(2016) can strictly be achieved by femtosatellites. The mission requires 100 's $-1,000$ 's of spacecrafts each measuring $4 \times 4 \times 4.25 \mathrm{~cm}$, with,

1 a digital microthruster system of total power $=1.6 \mathrm{~W}$ and total mass $=95.5 \mathrm{~g}$.

2 miniaturised warm gas hydrazine system (total power $=1.7 \mathrm{~W}$ and total mass $=104.7 \mathrm{~g})$.

On the other hand, Wu et al. (2014) cite short lead time, simplicity, relatively lower costs, batch production and minimal financial loss in case of failure as small satellite attributes that uniquely enable that mission. Because large spacecraft tethers possess complicated deployment and braking mechanisms, Carlson and Nakamura (2000) propose a small satellite tethered mission that can achieve simpler tether deployment and braking mechanisms due to their smaller sizes. Moreover, modularity and a 'plug-and-play' electrical bus synonymous with small satellites will allow common interface of all parts and frequent change-out of parts.

Innovative and agile systems engineering practices identified with formulating and implementing small satellite missions spawns synergies that empower formation flying missions. Notably, the ingenious systems engineering practices ubiquitous in the body of literature were: batch production of identical spacecraft (Wloszek et al., 2013; Gill et al., 2013; Wu et al., 2014; Bonin et al., 2015; Carlson and Nakamura, 2000); use of commercial of the shelf (COT) components (Subramanian et al., 2015; Ben-Yaacov and Gurfil, 2013; Hadaegh et al., 2016; Gangestad et al., 2013; Wu et al., 2014; Bonin et al., 2015; Carlson and Nakamura, 2000); frequent use of derivative simple spacecraft designs (Underwood et al., 2015; Gangestad et al., 2013; Bonin et al., 2015); willingness to embrace risk by flying relatively low TRL components (Gill et al., 2013; Subramanian et al., 2015; Griesbach et al., 2013); and modularity approach in subsystems design (Underwood et al., 2015; Ben-Yaacov and Gurfil, 2013). Majority of the papers detailed the mission systems engineering analysis and design - characterising the trajectory architecture, required propellant, $\Delta V$, etc.

Moreover, missions described in Wloszek et al. (2013), Wu et al. (2014), Griesbach et al. (2013), Bonin et al. (2015) and Carlson and Nakamura (2000) further included the spacecraft subsystems breakdown describing the constituent hardware parts in broad engineering detail.

Payload instruments engineering design analysis, trade-offs and sizing is also highlighted in a couple of reviewed missions. For instance, the Fourier transform spectrometer (FTS) is analysed in Wloszek et al. (2013); InSAR X-band transmitter is dissected in Peterson et al. (2008); analysis of geolocation via time difference of signal arrival is conducted in Leiter and Gurfil (2013); and, distributed configuration of a space telescope is scrutinised in Carlson and Nakamura (2000). Though it may appear that capabilities of small spacecraft are likely to be stifled by their relatively small size, innovative mission implementation practices such as formation flying mitigate this potential drawback. The review further observed that several missions had dual objectives, for instance the QB50 mission (Gill et al., 2013) had a formation flying technology demonstration objective, and a scientific objective - to investigate the thermosphere. Moreover, the formation in $\mathrm{Wu}$ et al. (2014) has a scientific objective to monitor polar sea ice in addition to demonstrating formation flying technologies. With the exception of Peterson et al. (2008), Hadaegh et al. (2016) and Leiter and Gurfil (2013), all the reviewed missions employ cubesats. The prevalence of this class 
of nanosatellites can be explained by the established basic form factor standard of $1 \mathrm{U}$ $(10 \times 10 \times 10 \mathrm{~cm})$ that conforms to the entrenched Poly Picosatellite Orbital Deployer (P-POD) deployment system.

The bulk of missions reviewed was composed of homogeneous formations that augur well for innovative systems engineering practices such as batch production. Heterogeneous formations were however used in three missions. A small spacecraft was part of the formation with two large spacecraft forming InSAR instrument in Peterson et al. (2008); one $3 \mathrm{U}$ cubesat is in formation with two $2 \mathrm{U}$ cubesats in $\mathrm{Wu}$ et al. (2014); and, the formation in Underwood et al. (2015) consists of two $3 \mathrm{U}$ cubesats and a single 15U microsatellite.

Based on the number of reviewed individual research publications, technology demonstration missions dominated the body of reviewed missions at $67 \%$ followed by earth observation $20 \%$ and lastly space exploration $13 \%$. This trend is not surprising because spacecraft formation flying is still primarily an active area of research - hence the plethora of technology validation missions.

\subsection{Future directions}

Initial formation acquisition and reconfiguration are two critical areas that conspicuously received little or no attention from the reviewed literature. The reviewed research focused on the guidance and control regimes required to maintain already acquired formations, e.g., Scharf et al. (2003b, 2004), Inalhan et al. (2002), Gill et al. (2013), etc. However, initialisation and reconfiguration of the formation geometrical structure is not a trivial problem to characterise or solve. Though this topic has received some attention from publications such as, Scharf et al. (2003a), Schlanbusch et al. (2011), Zhang and Duan (2015), Walls et al. (2005), Yang et al. (2003), etc. more research is required. Moreover, the issue of formation initialisation and reconfiguration is further exacerbated in small satellite formations because such formations are more propellant-constrained than larger monolithic spacecraft. Therefore, more research effort is required to formulate optimal guidance and control frameworks that are thrust free or require minimum actuation to initialise and/or reconfigure a formation.

Though a couple of missions, e.g., Underwood et al. (2015), Wu et al. (2014) and Griesbach et al. (2013) propose to demonstrate inter-satellite communication. Further research into inter-satellite communication protocols and hardware of low-mass and low-power is needed. Moreover, establishing an IEEE standard governing inter-spacecraft communication for formation flying would be a worthwhile research effort. Establishing such a standard would also invigorate innovative systems engineering practices synonymous with small satellite formation flying.

The paucity of research publications tackling spacecraft collision avoidance among formation members (e.g., Lee et al., 2015) indicates that additional investigation is required in this field. Although ambient orbital dynamics render collision unlikely during normal formation configurations; spacecraft malfunction or other external factors may disrupt the formation configuration enough to cause members to collide. Therefore, strategies to either mitigate or avoid collision entirely should be researched. A swarm mission (Hadaegh et al., 2016) would immensely benefit from such research because swarm members are more vulnerable to collisions due to their vast numbers.

Finally, research on how to achieve low-mass, low-power small satellite subsystems hardware components in general should continue in earnest. Priority can be accorded 
to propulsion and power subsystems because they constitute the most contribution to spacecraft mass. Devising low-power components for other spacecraft subsystems, e.g., attitude determination and control, guidance navigation and control, command and data handling, etc. will similarly promote lower spacecraft mass.

\section{Conclusions}

After scrutinising several missions with the aim of establishing if small satellites uniquely empower spacecraft formation flying missions, the main finding of this review arrives at the same conclusion as Chung et al. (2016) - small satellites do uniquely empower spacecraft formation flying missions. According to Chung et al. (2016), small satellites are enabling multi-satellite missions that were not otherwise possible because of their small size and modular nature. In this review, we have established findings that provide evidence in support of our hypothesis. For instance some of the main unique attributes of small satellites that foster spacecraft formation flying (see Subsection 3.1) include:

- Small satellites exclusively permit design of instruments to be deployed in a formation, e.g., Wloszek et al. (2013), Peterson et al. (2008) and Plice et al. (2019).

- Because of their size - small satellites uniquely undertake missions that are impossible or unsuited for large monolithic platforms, e.g., spacecraft swarm (Hadaegh et al., 2016; Plice et al., 2019), in-situ atmosphere study (e.g., Gill et al., 2013), and differential drag controlled formations (e.g., Ben-Yaacov and Gurfil, 2013).

- Innovative systems engineering practices uniquely advanced by small satellites such as batch production immensely enable expeditious implementation of formation flying missions.

- Simplicity and relatively lower costs of small satellite are consistent with desired properties of nodes in a multi-agent system. Consequently, by possessing these attributes, small satellites further uniquely empower formation flying missions (multi-agent system).

Though only two missions have been successfully flown in space Bonin et al. (2015) and Gangestad et al. (2013); spacecraft formation flying continues to mature as a discipline. The plethora of proposed missions is indicative of this upward trend and small satellites are uniquely positioned to empower this burgeoning field.

\section{Acknowledgements}

The author acknowledges the guidance accorded by Dr. Stephanie Gil from the School of Computing Informatics and Decision Systems Engineering, Arizona State University. 


\section{References}

Ben-Yaacov, O. and Gurfil, P. (2013) 'Long-term cluster flight of multiple satellites using differential drag', Journal of Guidance, Control and Dynamics, Vol. 36, No. 6, pp.1731-1740.

Bonin, G., Roth, N., Armitage, S., Newman, J., Risi, B. and Zee, R. (2015) 'CanX-4 and CanX-5 precision formation flight: mission accomplished!', Proceedings of the 29th Annual AIAA/USU Conference on Small Satellites.

Cannon, H., Sanchez, H. and Mcintosh, D.M. (2018) 'Starling1 - mission technologies overview', NASA Scientific and Technical Information, ARC-E-DAA-TN59780, Document ID: 20180007374 (Acquired 21 November 2018).

Carlson, J. and Nakamura, Y. (2000) 'The Kyushu/US Experimental Satellite Tether (QUEST) Mission, a small satellite to test and validate spacecraft tether deployment and operations', Proceedings of the 14th Annual AIAA/USU Conference on Small Satellites.

Chung, S., Bandyopadhyay, S., Foust, R., Subramanian, G. and Hadaegh, F.Y. (2016) 'Review of formation flying and constellation missions using nanosatellites', Journal of Space and Rockets, Vol. 53, No. 3, pp.567-578.

Gangestad, J., Hardy, B. and Hinkley, D. (2013) 'Operations, orbit determination, and formation control of the AeroCube-4 CubeSats', Proceedings of the 27th Annual AIAA/USU Conference on Small Satellites.

Gill, E., Sundaramoorthy, P., Bouwmeester, J., Zandbergen, B. and Reinhard, R. (2013) 'Formation flying within a constellation of nano-satellites: The QB50 mission', 6th International Workshop on Satellite Constellation and Formation Flying, Acta Astronautica, Vol. 82, No. 1, pp.110-117.

Griesbach, J., Westphal, J., Roscoe, C., Smugeresky, J., Hawes, D. and Carrico, J. (2013) 'Proximity operations nano-satellite flight demonstration (PONSFD) rendezvous proximity operations design and trade study results', Proceedings of the Advanced Maui Optical and Space Surveillance Technologies Conference, Maui, HI.

Hadaegh, F.Y., Chung, S.J. and Manohara, H.M. (2016) 'On development of 100-gram-class spacecraft for swarm applications', IEEE Systems Journal, Vol. 10, No. 2, pp.673-684.

Inalhan, G., Tillerson, M. and How, J. (2002) 'Relative dynamics and control of spacecrafts formations in eccentric orbits', Journal of Guidance Control and Dynamics, Vol. 25, No. 1, pp.48-59.

Lee, D., Sanyal, A.K. and Butcher, E.A. (2015) 'Asymptotic tracking control for spacecraft formation flying with decentralized collision avoidance', Journal of Guidance, Control, and Dynamics, Vol. 38, No. 4, pp.587-600.

Leiter, N. and Gurfil, P. (2013) 'Real-time geolocation with a satellite formation', Proceedings of the 27th Annual AIAA/USU Conference on Small Satellites.

National Aeronautics and Space Administration (1957) Sputnik 1: Nasa Space Science Data Coordinated Archive (NSSDCA) Master Catalog [online] https://nssdc.gsfc.nasa.gov/nmc/ spacecraftDisplay.do?id=1957-001B (accessed 3 March 2018).

Peterson, E., Zee, R. and Fotopoulos, G. (2008) 'Possible orbit scenarios for an InSAR formation flying microsatellite mission', Proceedings of the 22nd Annual AIAA/USU Conference on Small Satellites, Vol. SSC08-VI-5.

Plice, L., Dono, A. and West, S. (2019) 'Helioswarm: swarm mission design in high altitude orbit for heliophysics', NASA Scientific and Technical Information, ARC-E-DAA-TN72306, Document ID: 20190029109 (Acquired 20 August 2019).

Scharf, D., Ploen, S., Hadaegh, F., Keim, J. and Phan, L. (2003a) 'Guaranteed initialization of distributed spacecraft formations', AIAA Guidance, Navigation, and Control Conference and Exhibit.

Scharf, D.P., Hadaegh, F.Y. and Ploen, S.R. (2003b) 'A survey of spacecraft formation flying guidance and control. (Part I): guidance', Proceedings of the American Control Conference, Vol. 2, pp.1733-1739. 
Scharf, D.P., Hadaegh, F.Y. and Ploen, S.R. (2004) 'A survey of spacecraft formation flying guidance and control. (Part II): Control', Proceedings of the 2004 American Control Conference, Vol. 4, pp.2976-2985.

Scharf, D., Hadaegh, F. and Ploen, S. (2005) 'Precision formation delta-v requirements for distributed platforms in earth orbit', International Society for Optical Engineering, Vol. 5659, No. 16, pp.312-323.

Schlanbusch, R., Kristiansen, R. and Nicklasson, P.J. (2011) 'Spacecraft formation reconfiguration with collision avoidance', Automatica, Vol. 47, No. 7, pp.1443-1449.

Sholomitsky, G., Prilutsky, O. and Rodin, V. (1977) 'Infra-red space interferometer', Proceedings of the 28th Int. Astro. Fed. Congress, Paper IAF-77-68.

Subramanian, G.P., Fousty, R., Chen, D., Chan, S., Rogers, Y.T.D., Kokkat, J., Morgan, S.B.D., Chung, S-J. and Hadaegh, F.Y. (2015) 'Information-driven systems engineering study of a formation flying demonstration mission using six cubesats', Proceedings of the 53rd AIAA Aerospace Sciences Meeting, Paper 2015-2043, AIAA.

Underwood, C., Pellegrino, S., Lappas, V.J., Bridges, C.P. and Baker, J. (2015) 'Using CubeSat/micro-satellite technology to demonstrate the autonomous assembly of a reconfigurable space telescope (AAReST)', Acta Astronautica, Vol. 114, pp.112-122.

Walls, J., Howard, A., Homaifar, A. and Kimiaghalam, B. (2005) 'A generalized framework for autonomous formation reconfiguration of multiple spacecraft', 2005 IEEE Aerospace Conference, pp.397-406.

Waswa, P.M. and Redkar, S. (2017) 'A survey of space mission architecture and system actualisation methodologies', International Journal of Space Science and Engineering, Vol. 4, No. 3, pp.234-252.

Wloszek, P., Glumb, R., Lancaster, R., Lietzke, C., McCarty, S., Arlas, J., Heidt, B., Ramirez, M. and Singh, V. (2013) 'FTS CubeSat constellation providing 3D Winds', Proceedings of the 27th Annual AIAA/USU Conference on Small Satellites.

Wu, S., Mu, Z., Chen, W., Rodrigues, P., Mendes, R. and Alminde, L. (2014) 'TW-1: a cubesat constellation for space networking experiments', Proceedings of the 6th European CubeSat Symposium.

Yang, G. Kapila, V. and Wong, H. (2003) 'Fuel optimal initialization of a spacecraft formation', 42nd IEEE International Conference on Decision and Control (IEEE Cat. No. 03CH37475), Vol. 4, pp.3591-3596.

Zhang, S. and Duan, H. (2015) 'Gaussian pigeon-inspired optimization approach to orbital spacecraft formation reconfiguration', Chinese Journal of Aeronautics, Vol. 28, No. 1, pp.200-205. 